\title{
SALUTO DEL PRESIDENTE DELL'ISTITUTO LOMBARDO
}

\author{
GIANPIERO SIRONI (*)
}

Sono molto lieto di rivolgere un saluto di benvenuto ai relatori ed a tutti i partecipanti.

Desidero ringraziare il Presidente della Repubblica, Giorgio Napolitano, che ha concesso il suo Alto Patronato al Convegno odierno, e ringraziare altresì il Ministero per i Beni e le Attività Culturali, il Comune di Milano e l'Accademia dei Lincei per il loro patrocinio.

Desidero ringraziare il Teatro alla Scala per avere scelto di collaborare con l'Istituto Lombardo nell'organizzare questo Convegno e di ospitarne lo svolgimento.

Mi piace sottolineare che non accade frequentemente all'Istituto Lombardo di organizzare un evento, come quello di oggi, congiuntamente con una istituzione, come il Teatro alla Scala, la cui origine (1778) precede quella del nostro Istituto (fondato nel 1797, localizzato a Milano dal 1810).

Entrambe le Istituzioni, oltre ad essere state attive per tutto il tempo dalla loro origine, lo sono state in particolare nel periodo, l'Ottocento, che ha visto essere attivi i due Grandi di cui il Convegno odierno tratterà, in occasione del bicentenario della nascita (ricordo altresì che Giuseppe Verdi morì il 27 gennaio 1901; quindi tra due giorni cadrà l'anniversario della sua morte).

L'Istituto Lombardo, in particolare, ebbe nell'Ottocento un ruolo molto attivo nella cultura, intesa in senso lato, in particolare milanese e lombarda.

(*) Presidente dell'Istituto Lombardo Accademia di Scienze e Lettere, Milano. 
Non è nemmeno il caso che io ricordi, lo faranno certamente altri, gli intensi rapporti che Giuseppe Verdi ebbe con il Teatro Alla Scala.

L'Istituto Lombardo non ha avuto rapporti altrettanto diretti.

Mi piace però ricordare che Verdi ha composto una Messa da Requiem per la morte di Alessandro Manzoni (rappresentata il 22 maggio 1874 nella chiesa di San Marco, a un passo dalla sede del nostro Istituto) e che Alessandro Manzoni, nel 1859, quando la Lombardia venne liberata, fu acclamato Presidente dell'Istituto Lombardo; nomina subito confermata dal Re Vittorio Emanuele II.

Penso che questa menzione richiami alla mente di molti la valenza che Giuseppe Verdi ebbe nei riguardi delle vicende che condussero all'unità d'Italia, ma, di nuovo, non è certo il caso che sia io a parlare di questo.

Mi piace in ogni caso accostare il nome di Alessandro Manzoni a quello di Giuseppe Verdi; si tratta di due capisaldi della nostra cultura, vivi oggi come ieri nell'apprezzamento generale; Verdi per la musica; Manzoni per la lingua italiana.

Se a Giuseppe Verdi è dedicato, insieme a Richard Wagner, il convegno odierno, alla storia della lingua italiana abbiamo dedicato un convegno lo scorso 20 dicembre ed è attualmente in corso una mostra (fino al 1 marzo) nella sede dell'Istituto, a Palazzo Landriani, via Borgonuovo 25.

Desidero ringraziare i membri del Comitato scientifico che ha organizzato il Convegno e in particolare Luciano Martini, anche per avere procurato e reso disponibili le esecuzioni della NBC Orchestra, diretta da Arturo Toscanini, che saranno presentate nel pomeriggio di oggi.

Solitamente, in occasione di un Convegno dell'Istituto, do notizia di un evento successivo; permettetemi allora di cogliere l'occasione odierna per segnalare che l'Istituto Lombardo dedica l' adunanza che si terrà giovedì 31 gennaio prossimo alla commemorazione del Card. Carlo Maria Martini, che è stato membro effettivo dell'Istituto. La commemorazione sarà svolta da Mons. Franco Buzzi, Prefetto della Biblioteca Ambrosiana e membro effettivo dell'Istituto, e da Mons. Pier Francesco Fumagalli, VicePrefetto della stessa Biblioteca.

La commemorazione, alla quale il pubblico è invitato, si terrà alle ore 15,00 nella sala delle adunanze dell'Istituto, nel Palazzo di Brera (via Brera 28).

In quella stessa occasione il prof. Giulio Guderzo presenterà il primo volume del carteggio del Card. Giovan Battista Montini, Papa Paolo VI, pubblicato di recente dall'Istituto Paolo VI.

Auguro a tutti una proficua partecipazione al Convegno. 